\title{
LA FISIOGNOMÍA FEMENINA A TRAVÉS DEL MANIFIESTO DE LOS SECRETOS DEL COITO DE AL-SHAYZARİ (XIII)
}

\author{
Miguel Ángel Lucena Romero \\ Universidad de Málaga \\ mlucena@uma.es
}

\begin{abstract}
RESUMEN
En cualquier sistema teocrático y monoteísta, la imagen femenina se representa como una entidad sexual, reducida a su dimensión más material y física, soslayando cualquier peculiaridad intelectual o psicológica. El objetivo del siguiente artículo es describir y analizar la categorización, sin recato alguno, de las mujeres según su aptitud sexual y, para ello, nos centramos en el texto fisiognómico legado por el tratadista sirio al-Shayzarī (s. XIII) en su compendio sexual Kitäb al-İdäh fi asrär al-nikäh (El Manifiesto de los secretos del coito). Tras una breve introducción al concepto árabe e islámico de fisiognomía, analizamos la línea metodológica que sigue nuestro autor, a saber, el método expresivo, étnico y zoológico, cuando se refiere a la fisiognomía femenina.
\end{abstract}

Palabras Clave: Fisiognomía, sexualidad, Edad Media, mujeres, islam.

\section{THE FEMALE PHYSIOGNOMY THROUGH ELUCIDATION OF THE SECRETS \\ OF LAWFUL INTERCOURSE OF AL-SHAYZARİ (XIII)}

\begin{abstract}
In any theocratic and monotheistic system, the female image is represented as a sexual entity, reduced to its most material and physical dimension, avoiding any intellectual or psychological peculiarity. The objective of the following article is to describe and analyze the categorization, without any modesty, of women according to their sexual aptitude and, for this, we focus on the physiognomic text bequeathed by the Syrian writer al-Shayzarī $\left(13^{\text {th }}\right.$ century) in his compendium sexual Kitäb al-Ị̇äh fì asrär al-nikäh (Elucidation of the secrets of lawful intercourse). After a brief introduction to the Arabic and Islamic concept of physiognomy, we analyze the methodology that our author follows, namely, the expressive, ethnic and zoological method, when referring to female physiognomy.
\end{abstract}

KEYwORDS: Physiognomy, sexuality, Middle Ages, women, Islam.

La representación del cuerpo femenino en cualquier literatura constituye un universo poético. Cada discurso recrea sus cuerpos, según sus preferencias, orígenes y propósitos. En ella se manifiestan los valores y tabúes de cada civilización, 
aunque a veces esta representación se vea estereotipada por ciertas reflexiones propias de la sociedad. De ahí que, en sistemas teocráticos como el islámico, la imagen femenina no tiene un lugar real en la literatura y estas son descritas por su físico, su rostro o como un ente somero, «añádase que el olor de la cortesana es más fragante; sus ropajes, más perfumados; su andar, más delicado; y el timbre de su voz más frágil. Por ello, resulta más propicia a los corazones, por no hablar de que puedes tomarla por delante y por detrás, como mejor te guste y siempre por lo lícito» (Al-Yáhiz, 2018: 42). Esta cosificación, en cambio, contrastaría con la literatura árabe actual, en la que la mujer piensa y goza: "Así que pronto supe lo que quería: una mente despierta en un cuerpo despierto. Lo supe incluso antes de leer, en los libros eróticos árabes que me son tan queridos, ideas que sustentaran mi tesis» (al-Neimi, 2012: 12).

$\mathrm{El}$ interés de los literatos por el cuerpo femenino, planteado como motivo y perspectiva falocéntrica para satisfacer las necesidades masculinas, ha sido un tema harto común en las fuentes clásicas árabes desde los primeros tiempos. A la sazón, su representación se ha situado al nivel de la religión, ya que «el conocimiento del cuerpo humano suponía, al mismo tiempo, un reconocimiento a la grandeza creadora de Dios» (Álvarez de Morales, 1998: 129). Es más, la reflexión que engloba todo lo relativo al estudio del cuerpo se ha desarrollado en la literatura islámica desde distintos puntos de vista: religión, poesía, medicina, derecho, administración e incluso en las fuentes jurídicas (Tena, 2008: 45-61).

En este sentido, las fuentes clásicas árabes e islámicas de índole fisiognómica otorgan de manera directa un razonamiento sobre la naturaleza y esencia del cuerpo humano, a la vez que son un índice certero para conocer la dimensión del cuerpo femenino en la época medieval islámica ${ }^{1}$. Así pues, el objetivo principal del siguiente artículo es describir y analizar la categorización, sin recato alguno, de las mujeres según su actitud y potencialidad en el coito y, para ello, nos centramos en el texto fisiognómico legado por el tratadista sirio al-Shayzarī (s. XIII) en su compendio sexual Kitäb al-Ị̇äh fì asrär al-nikäh (El Manifiesto de los secretos del coito). Tras una breve introducción al concepto árabe e islámico de fisiognomía, analizamos la línea

${ }^{1}$ En el terreno de la fisiognomía medieval destacan indudablemente las extraordinarias aportaciones de la investigadora italiana Antonella Ghersetti a los estudios árabes e islámicos. La experta en este ámbito de estudio ha dedicado gran parte de su erudición a la materia fisiognómica medieval y ha estudiado y comparado obras de autores clásicos como Ibn Isḥāq, al-Rāzī, Ibn 'Arabī, al-Dimashqī, entre otros. En su legado científico resalta además su última investigación sobre la representación fisiognómica de las esclavas en el siglo XIV. Asimismo, en su recorrido científico, Antonella Ghersetti ha traducido al italiano uno de los tratados aristotélicos más representativos en esta temática, como fue el Kitab Aristạtalìis al-fayläsüffi l-firāsa (traducido a su vez al árabe por el célebre Ibn Isḥāq) e igualmente otra de sus contribuciones más excepcionales se encuentra en la traducción al italiano de la obra dedicada en exclusiva a la compra-venta de esclavas, Risälat ŷami at li-funūn nāfía fi shirā' al-raqìq wa-taqlìb al-áaid, del médico cristiano Ibn Buțlān (s. XI). 
metodológica que sigue nuestro autor, a saber, el método expresivo, étnico y zoológico, cuando se refiere a la fisiognomía de las mujeres. Este estudio sirve además para explorar el canon de belleza femenino establecido por los árabes entre la época abasí y la mameluca.

\section{AL-SHAYZARİ Y EL MANIFIESTO DE LOS SECRETOS DEL COITO}

En el corpus literario de al-Shayzarī se encuentra uno de los fragmentos más completos sobre fisiognomía femenina. En cuanto al autor, como bien indica su gentilicio, a este se le sitúa en Shayzar, en la actual Siria, al noroeste de Hamā, a las orillas del río Orontes (al-Rūmī, 2007: 383-384). Su fecha de muerte es algo más imprecisa. Los investigadores y enciclopedistas proponen dos fechas de muerte bien distintas, siglo XII y XIV. Sin embargo y después de un largo análisis biobibliográfico sobre el autor, deberíamos colocar su vida durante el siglo XIII, sin descartar la posibilidad de que muriera a lo largo del primer cuarto del siglo XIV².

Según consta en los repertorios biobibliográficos, al-Shayzarī redactó, además de El Manifiesto de los secretos del coito, otras seis obras más, dos de ellas perdidas, al-Tuhfa wa-l-turfa y al-Hadā'iq wa-l-thimär fì nawädir al-qud̄at wa-l-buhalä, y ninguna de todo su corpus literario responde a la temática erótica. Por un lado, este dedicó parte de su erudición a la temática político-social como bien se refleja en al-Manhây al-maslīk fi sìyasat al-mulük y Nihäyat al-rutba al-zarifa fì talab al-hisba al-sharifa. Por otro lado, este compiló una obra dedicada a la interpretación de los sueños, Juläsat al-kaläm fì tä wöl al-ahläm, y un poemario consagrado al amor medieval, Rawdat al-qulüb wa nuzhat al-muhibb wa-l-mahbüb.

En lo que a la obra objeto de este estudio se refiere, esta se enmarca entre la etapa final del período abasí e inicios del imperio mameluco, caracterizados estos por su diversidad social, miscelánea y variada y, sobre todo, por la influencia de la cultura foránea, persa y turca, en la sociedad islámica. El Manifiesto de los secretos del coito viene a sumarse al amplio corpus bibliográfico sobre la erótica árabe en general. Esta obra es una compilación principalmente de afrodisíacos en la que hallamos uno de los capítulos más extensos sobre fisiognomía femenina, lo cual ha valido a su autor cierta popularidad y reconocimiento.

El contenido erótico de la obra se divide en dos secciones, una dedicada a los hombres y otra a las mujeres. En esta estructura, al hombre se le otorga el papel principal en el acto sexual, mientras que el objetivo en la sección femenina es doble:

${ }^{2}$ A esta conclusión hemos llegado después de varios años de estudio en los que hemos analizado su corpus bibliográfico y en especial su obra en materia erótica. En esta última, el autor evidencia entre sus fuentes referidas la fecha en la que posiblemente desarrolló su vida. Para una información más detallada, véase la introducción al autor de Lucena (2019: 135-152). 
el primero, embellecer el cuerpo de las mujeres con remedios y cosméticos y, el segundo, conocer y someter su disposición sexual. Así, el concepto de sexualidad acuñado por al-Shayzarī, compartido con el resto de escritores árabes, deja patente la posición social inferior de las mujeres, objeto pasivo al que hay que aderezar para servir y satisfacer al hombre.

Por último, cabe mencionar las relaciones intertextuales de nuestro autor con sus predecesores y contemporáneos. Según se menciona en El Manifiesto, las fuentes médicas principales de al-Shayzarī fueron las de Galeno, al-Rāzī, Avicena, Avenzoar y al-Ṭūsī, entre otras, mientras que en el ámbito fisiognómico parece haber

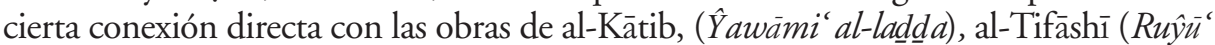
al-shaykh ilà șibä-hu fì l-quwwa 'alà al-bäh), pues al parangonarlas con El Manifiesto encontramos textos cuasi calcados. Asimismo, y aunque no existan evidencias textuales, pensamos que El Manifiesto ha influido de manera directa en el tratado fisiognómico Kitäb al-siyāsa fì 'ilm al-firāsa de al-Dimashqīî

\section{BREVE CONCEPTO DE FISIOGNOMÍA O FIRĀSA}

La literatura fisiognómica ocupa una posición en la que transcurren numerosos campos de investigación, como el sociológico y médico, pasando por los estudios de género, política, astrología, zoología o psicología. Esto hace que los tratados fisiognómicos transciendan de manera fehaciente en el islam clásico, debido a su carácter científico y a su finalidad empírica en la sociedad. Toda esta tradición no surgirá de la nada, sino que es un espejismo de las narraciones griegas, que han evolucionado desde la llegada del islam y, sobre todo, desde la instalación de los abasíes en el poder islámico.

Antes de comenzar, conviene mencionar sucintamente las principales acepciones que se han recogido sobre el término firäsa en los compendios lexicográficos árabes e islámicos. De la raíz $f-r-s$, en su forma quinta, tafarrasa, significa "escrutar", "notar algo de alguien" "clavar la mirada", "observar atentamente" o "descubrir una cualidad de alguien". La expresión firäsat bi-l-'ayn o firäsat bi-l-nažr significa literalmente "percepción intuitiva", "percepción del carácter, condición, estado o circunstancias a través del examen de las señales externas".

En la obra pseudo-aristotélica de Ḥunayn Ibn Isḥāq (m. 768) Kitāb al-firāsa (Libro de fisiognomía), se encuentra una de las primeras definiciones sobre fisiognomía. Según este, en un análisis fisiognómico de un individuo existe una estrecha relación entre las facultades intelectuales y los movimientos corporales, y estos se pueden ver modificados o alterados por estados externos como la embriaguez o el amor.

${ }^{3}$ Sobre la cuestión de las relaciones intertextuales de textos fisiognómicos de esta época profundiza la experta Antonella Ghersetti (2018: 21-45) en su reciente estudio sobre la obra de al-Dimashqī. 
En este sentido, la fisiognomía no solo sirve como método natural para conocer las actitudes humanas, sino que también son un indicador de las condiciones pasionales (Ghersetti, 1999: 51-55).

En cuanto a su clasificación en la literatura árabe e islámica, será el médico y filósofo Ibn Sīnā (m. 1037), conocido como Avicena, en su Risäla fì taqsìm al-úlūm al-'aqliyya (Epistola sobre la división de las ciencias mentales) quien registre la ciencia fisiognómica como una ciencia natural subalterna, situada entre la medicina, la astrología y la ciencia de la interpretación de los sueños (Anawati, 1976: 64-65). Casi dos siglos más tarde, Fajr al-Dīn al-Rāzī (m. 1209) sostiene en su Kitāb al-firāsa (Libro de fisiognomía) la posibilidad de practicar el método fisiognómico a través del uso de la teoría humoral. De esta manera, el aspecto exterior y el carácter interior de un individuo están estrechamente conectados y, por esta razón, al observar uno de los dos se podrá conocer también el carácter del otro (Mourad, 1939: 4).

En el diccionario de Ibn Manz̄ūr (m. 1312), Lisān al-'́arab (La lengua de los árabes), firäsa se define como "el hecho de escrutar u observar atentamente a un individuo", y en un sentido religioso se podría interpretar como "mirar con los ojos de Dios". Ya en época otomana, en la enciclopedia legada por Ḥāŷŷȳ Jalîfa (m. 1657), este define la fisiognomía como la ciencia a través de la cual se entiende a los individuos por su aspecto físico mediante un proceso inductivo de observación (Jalífa, 1964: 388). De este modo, el concepto que engloba el término firäsa se debe relacionar también con el objeto de percibir la inteligencia y todo aquello inaccesible a los sentidos (Ghersetti, 1994: 16).

Llegados a este punto, podemos afirmar que en la literatura fisiognómica se plasma un proceso inductivo para examinar el cuerpo humano con un fin determinado, el de conocer el físico no visible y la conducta de un individuo a través de algunas señales corporales. Deducimos pues que el beneficio de esta práctica permite analizar el carácter y la actitud de los seres humanos y es útil, por tanto, para conocer la personalidad de los cónyuges, amigos, esclavos, políticos, etc., en una sociedad en la que las relaciones humanas se encuentran aparentemente muy limitadas.

Con estas definiciones y en pos de situar la fisiognomía en un marco determinado, esta la colocamos como una rama del saber que encuentra su origen en el legado griego, y cuyo eje no está sujeto ni a las teorías médicas, ni a la filosofía, ni al arte, aunque se pueda incluir en estas. Esta, más bien, constituye una "pseudociencia" que no conlleva resultados empíricos, como es el caso de la astrología, o teóricos, como las matemáticas (Cadden, 1993: 86). La experta en textos fisiognómicos Antonella Ghersetti afirma que "physiognomy shares the same position with medicine, having in common the same foundations of the natural sciences which are elaborated in the light of experience, with the effect that any criticism of the one will also apply to the other» (Ghersetti, 2007: 301). La posición de los tratados de corte fisiognómico en la clasificación de la erudición medieval se encuentra junto a obras de astrología, medicina, adivinación, quiromancia, religión, filosofía, interpretación de los sueños, entre otros. (Cadden, 1993: 186).

Sin embargo, observamos que el eje monotemático de toda esta erudición difiere según el género del que se trate, pues, normalmente, en el caso de los textos concernientes a la esfera masculina, la explicación de los signos exteriores informa 
sobre las características psicológicas, intelectuales, temperamentales, fisiológicas, entre otras, sin atender a los aspectos sexuales, mientras que el discurso de la fisiognomía femenina se centra exclusivamente en el mundo de lo sexual y la belleza corporal. El estudio de la fisiognomía femenina en los tratados árabes e islámicos queda relegado totalmente al servicio del eros y está estrechamente ligado al sexo, evitando o eludiendo cualquier referencia relativa a las capacidades intelectuales de las mujeres.

La relevante carga erótica que emana de la interpretación de las señales corporales en los textos de fisiognomía provoca que el tópico de la belleza femenina sea tratado sobremanera en la literatura medieval. Es a través de estos textos que se establecen los estereotipos de belleza femenina, siendo este el objetivo principal de dicho discurso para la compra-venta de esclavas:

Slaves are a variety of merchandise, subject to bargaining and chaffering over price; and both vendor and purchaser need to examine the piece of goods carefully, and subject it to a close scrutiny. For this purpose, there is a requisite the same sort of ocular selection as is obligatory in relation to all commodities sold. If a thing cannot be priced by measure of capacity, weight, number or linear measurement, it is judged by beauty or ugliness. Of this the only judge is a person with a trained eye, skillful in his trade (al-Ŷăhiz, 1980: 24-25).

Por ende, entendemos que los tratados de fisiognomía femenina se caracterizan por ilustrar pedagógicamente el procedimiento para la adquisición de mujeres, sirviendo estos de manual de apoyo y consejo para los compradores de esclavas. Estos informan, de manera apodyópsica, previamente a la realización de la compra, de la forma de sus órganos genitales, sus partes del cuerpo y de las diferentes actitudes sexuales que estas muestran antes y durante el acto carnal. Por esta razón y según la misma Antonella Ghersetti, el ejercicio de observación de la fisiognomía natural de un individuo a través de sus rasgos físicos, preferentemente faciales, con el objetivo de conocer su anatomía y su psicología, traslada esta disciplina a la práctica médica de examinar a las esclavas para conseguir una compra eficaz:

These constitute an important zone of intersection within scientific writing of firāsa. Medical and physiognomic knowledge reinforce each other in the clinical and psychological examination of the slave. Verification of "value for money" was a not simple matter of ascertaining whether the slave had a sound state of health. It extended to aspects of his personality and aptitude for the tasks required, and this of course came within the field of firasa (Ghersetti, 2007: 293).

Así, por lo general, las características más valoradas de las esclavas según las fuentes fisiognómicas árabes e islámicas fueron: la armonía en el acto conyugal, la predisposición sexual, las vaginas limpias, estrechas y grandes, las esclavas de buen gobierno y orgasmo, bellas y lascivas. Con todo esto, el deseo divino de los hombres "casaos con las mujeres que os gusten, dos, tres o cuatro» (Corán, 4:3) por conseguir, de la manera que fuera, una buena esposa se convierte en un tópico recurrente en este tipo de fuentes, pese a que no se mencione explícitamente. Existe un sinfín de cuentos que relatan la relación pasional de hombres con sus esclavas, su necesidad de comprarlas y las hazañas vividas con ellas. 


\section{CATEGORIZACIÓN DE LAS MUJERES SEGÚN EL MANIFIESTO}

Ya en la introducción de la sección femenina de El Manifiesto se evidencia el objetivo principal para estas: «consideramos mencionar en esta segunda parte algo de los secretos de las mujeres, aquello por lo que incita a los hombres a copular con ellas y les obliga a inclinarse a ellas, razón por la cual son amadas por sus maridos» (al-Shayzarī, 2020: 71). En esta línea, el autor dedica toda su erudición a describir a capite ad calcem las partes del cuerpo femenino más deseadas por los hombres y los métodos más útiles para conocer su actitud sexual.

Según al-Shayzarī, en el canon de belleza femenino destacan las siguientes características: el color negro del pelo y de la mirada, el color rojo de la lengua, de las nalgas, de las mejillas y de las caderas, la redondez del cuerpo, el buen olor corporal, la anchura de ojos y pechos, las vaginas estrechas y limpias y las bocas, manos y pies pequeños (al-Shayzarī, 2020: 73). A partir de aquí, en la obra se despliegan siete capítulos protagonizados por tintes para el pelo, perfumes para todas las partes del cuerpo y recetas para engordar o estrechar los miembros deseados:

Sabed que el parto y el coito frecuente dilatan la vagina, de modo que desaparece el placer natural. Es necesario que esto se evite a través de los remedios que a continuación mencionamos:

Receta de un medicamento que estrecha la vagina: se coge piel de opopónaco quemado, pezuñas de cabra y burro quemados, métel quemado, cebada silvestre, de cada uno un dírham, se tritura todo fino y se amasa con aceite de behen y se deja. Luego, la mujer se pondrá el peso de un daniq de esto, tres veces al mes, una vez cada diez días, pero no hará esto cuando esté menstruando. Se queman los simples para que puedan ser triturados sin excederse, pues ciertamente se estrecha la vagina hasta quedar como una mujer virgen (Shayzarī, 2020: 105-106).

Una vez dispuesto y acicalado el cuerpo de las mujeres, en esta obra se descubren, mediante una serie de pautas, las diferentes actitudes sexuales femeninas en el capítulo titulado "De las señales que guían hacia las vulvas de las mujeres sobre el exceso o escasez de deseo y otros asuntos, a través de la fisiognomía». En este, se detallan todos los aspectos a tener en cuenta a la hora de elegir una esposa o comprar una esclava y, para alcanzar su objetivo, al-Shayzarī sigue la triple metodología aristotélica (Armstrong, 1958: 52-56):

- Método expresivo. Consiste en extraer los signos del carácter humano a través de las expresiones emotivas. Por ejemplo, la expresión del rostro cuando el individuo está enfadado o tiene una preocupación. O bien, cuando a través de las facciones se puede conocer la actitud del individuo.

- Método étnico. Consiste en clasificar a los seres humanos según su procedencia étnica, racial y geográfica. Este método sirve para organizar la disposición y el valor de los esclavos con el motivo de su compra-venta.

- Método zoológico. Consiste en analizar la apariencia humana mediante un examen corporal lo que conlleva a un examen que evoca la semejanza de la persona con un animal. Un ejemplo es la comparación del elefante, caracterizado por su docilidad e inteligencia, con el ser humano. Igualmente, este método sirve para describir y parangonar el tamaño de ciertas partes del cuerpo. 


\section{MÉTODO EXPRESIVO}

El primer método usado por al-Shayzarī en El Manifiesto es el expresivo. Este informa de las recomendaciones que se deben seguir para conocer las habilidades femeninas en el acto carnal, a través del examen de los signos del rostro y cuerpo: la intensidad del apetito sexual (shahwa), el deseo ( $r a g b a)$, la libido (shabaq) y la lascivia (gulma). Así, el análisis exhaustivo del cuerpo femenino desvelará literalmente la esencia sexual de las mujeres y esto facilita a su vez la capacidad de elecciónadquisición por parte de los compradores de esclavas con buena predisposición sexual y físico óptimo.

En el caso de la descripción de al-Shayzarī, las zonas más referenciadas se encuentran casualmente en la cara, la zona más visible: nariz, mentón, boca, orejas, ojos, lengua y mejillas. A partir de estas, se conoce la mayor parte de la información sobre la disposición sexual de las mujeres, mientras que, de las partes menos visibles como son nalgas, hombros y piernas, los datos son algo más escuetos:

Si la boca de la mujer es ancha su vagina también lo es, si su boca es pequeña su vagina es estrecha. Si sus labios son gruesos, los labios de su vagina son gruesos. Si su labio superior es más grueso los labios de su vagina son menudos. Si su labio inferior es pequeño su vagina es pequeña. Si su lengua es de color rojo intenso su vagina carece de humedad. Si su lengua está cortada por la punta su vagina es muy húmeda. Si es de nariz aguileña manifiesta poco deseo en el coito. Sin embargo, si el lóbulo de sus orejas está separado, esto pone de manifiesto un intenso deseo de coito [...] si la mujer cuenta con pies y manos carnosos su vulva es enorme y tiene el favor de su marido. Si la mujer tiene las piernas nobles y fuertes, esto indica que es fogosa e impaciente para el coito. [...] Si es de piel rojiza y de ojos azules, la mujer posee un gran apetito sexual y si es risueña y ligera de movimiento, será igualmente muy lujuriosa.

[...] Los ojos alcoholados y grandes son indicativos de lascivia y estrechez de útero. La pequeñez de las nalgas y anchura de hombros indican el tamaño de la vagina, mientras que la inclinación del cuello hacia la espalda es indicativo del grosor de la vagina (Al-Shayzarī, 2020: 77).

En cuanto a otros signos del cuerpo que indican la tipología sexual femenina, al-Shayzarī distingue dieciocho categorías, siendo su argumento principal la edad y el tallaje. Por un lado, el autor recoge que las mujeres más lascivas son aquellas que ya completaron la etapa de la adolescencia o aquellas de tallaje alto; según este, la primera, «está completamente formada, es de buenos modales» y la segunda, "desea el coito"; mientras que las púberes, bajas de estatura, muy anchas de cuerpo y flácidas son las menos aconsejables para el matrimonio, ya que estas «no desean el sexo y no están dispuestas» $\mathrm{y}$ "solo les gusta abrazar, besar, burlarse, conversar, bromear, divertirse y el trasiego carnal» (al-Shayzarī, 2020: 80-81).

Por último, una vez que nuestro autor ha detallado la disposición sexual femenina, este aborda una minuciosa lectura de los distintos tipos de vaginas y divide a las mujeres en ocho categorías: la grasienta, la delgada, la estéril, la hueca, la carnosa, la de los labios, la abotagada y la profunda. Según este, todas estas vaginas son 
defectuosas y no convienen a los hombres si estos no cumplen una serie de características. A la grasienta le conviene un pene de tres puños, a la delgada un pene grueso y corto, a la estéril un pene grueso y de gran glande, a la hueca, aunque es difícil de satisfacer, le conviene un pene largo y grueso, a la carnosa un pene de rápida eyaculación, la de los labios un pene largo y fino, a la abotagada un pene duro y fuerte y a la profunda ninguno le satisface, excepto la vagina de otra mujer (al-Shayzarī, 2020: 78-79).

\section{MÉTODO ÉTNICO}

En segundo lugar, nuestro autor emplea el método étnico. Este método de asociación racial sirve principalmente para seccionar a los seres humanos en distintas categorías, según su procedencia. En este caso, los tratadistas, a través de su pericia y de la experiencia de autores precedentes, facilitan a los compradores de esclavos una herramienta efectiva y variada, a la hora de seleccionar al servicio doméstico y profesional. Así pues, si se trata de un manual fisiognómico en el que se especifican las condiciones masculinas de los esclavos, en este prevalecerán y se describirán cualidades tales como la fuerza, la perspicacia, la inteligencia o la obediencia, entre otras.

Sin embargo, cuando se aplica este procedimiento en el terreno de la fisiognomía femenina, Bouhdiba (1980: 239) lo ha definido con el término de "geoerotología", siendo su principal peculiaridad la de seccionar a las mujeres según su origen étnico con el único fin de descubrir las cualidades femeninas en el terreno de la cohabitación y el matrimonio, ambos primordiales en la tradición islámica. Como ocurre con el método expresivo, las características más valoradas son la belleza corporal, la disposición sexual y el apetito. Por consiguiente, se entiende que en estos textos los tratadistas subrayan y advierten de manera pormenorizada sobre las distintas condiciones a tener en consideración a la hora de comprar una esclava o esposa.

Con este propósito, al-Shayzarī describe a aquellas mujeres que más convienen a los hombres por su higiene, las bizantinas, por su belleza y perfume, las andalusíes, o por su experiencia y disposición sexual, las mequíes, y aquellas que, al contrario, no convienen por su mala moral e higiene, las turcas y mongolas, por su fealdad, antipatía y hediondez, las indias, las esclavonas y las africanas. Al final de esta categorización, este describe a las mujeres con mayor pericia sexual, casualmente, todas de origen árabe: mequíes, basoríes, alepinas y bagdadíes:

Sabed que las mujeres bizantinas son las más limpias de útero, las andalusíes son las más bellas de estampa, las más penetrantes de olor, las más elogiadas por su progenie y las de mejores úteros. Las turcas y mongolas son las más sucias de útero, las más rápidas de parto y las de peor moral. Las procedentes de Sind, de la India y las esclavonas son las más desagradables, las más feas, las más rencorosas, las más descerebradas, las de peor gobierno doméstico, las más hediondas y de útero sucio. [...] Las mequíes tienen la belleza perfecta y son las mejores copulando, las mejores de todas estas razas, a pesar de que estas no están dotadas del color de las demás. Las egipcias son las más lascivas y voluptuosas en el coito. Las alepinas son las que tienen el cuerpo más fuerte y los úteros más duros. Las de la costa y las damascenas 
son mujeres moderadas y equilibradas en el disfrute. Las bagdadíes son más atractivas que ningunas otras, las más sociables y las más dispuestas para el coito. Y quien quiera convivir, cohabitar y la buena conversación, tiene que elegir a las iraquíes. Quien quiera tener buenos hijos que elija a las persas y a las árabes pues son las que están dotadas de las mejores condiciones, de todas las descritas (Al-Shayzarī, 2020: 79).

\section{EL MÉTODO ZOOLÓGICO}

Desde tiempos pretéritos, la zoología ha sido un tema recurrente entre los eruditos árabes. Se ha estudiado desde la evolución de los animales, sus enfermedades, su descripción física, hasta su inteligencia y sus distintos métodos reproductivos, entre otros. Así, por ejemplo, al-Ȳạhiz (m. 869), conocido por su tratado Kitāb alHaywān (El libro de los animales), afirma que la coyunda en exceso apresura la vejez, basándose en la teoría de que los animales que se aparean con más asiduidad suelen envejecer antes. Es más, según este autor los animales menos longevos son los pájaros, ya que se entregan al coito con asiduidad y los más longevos son los mulos que, al contrario de los pájaros copulan en menos ocasiones (al-Ŷāhiz, 1998: 78)4.

Así pues y como no podría ser de otra manera, nuestro autor se sirve del método zoológico aplicado a la sexualidad para informar sobre la descripción y coincidencia en el tamaño de los órganos genitales de los cónyuges en el acto sexual. Este nos presenta, a modo comparativo, los diferentes tamaños del órgano sexual, masculino y femenino, y los divide en tres tamaños: grande, mediano y pequeño. A cada uno de ellos le asigna un animal. En el caso masculino, elefante, caballo y carnero representan los tamaños de mayor a menor de los órganos genitales y, en el caso femenino, elefanta, yegua y oveja. De esta manera, al-Shayzarī pretende advertir sobre los daños que se pueden llegar a sufrir durante el acto sexual si se da el caso de que los órganos sexuales de los cónyuges son desiguales en tamaño. Así, es fácil deducir que a un pene grande le conviene una vagina grande, como si un elefante copula con una elefanta, a un pene mediano le satisface una vagina mediana, es decir, cuando copulan caballo y yegua y, por último, a un pene pequeño le conviene una vagina estrecha, como pueden ser los casos del carnero y la oveja. En caso contrario, si en el acto carnal coinciden un pene grande y una vagina pequeña, como pueden ser el símil del elefante y la oveja, las consecuencias serán dolorosas y dañinas:

El primer tipo: es cuando se encuentran el elefante y la elefanta, el caballo y la yegua, el carnero y la oveja. Este es el culmen de la coincidencia y del placer completo.

El segundo tipo: es cuando se encuentran el elefante y la yegua, el caballo y la elefanta, el carnero y la yegua. En este caso, existe un placer intermedio.

${ }^{4}$ Según el mismo autor, de este razonamiento se deduce el motivo por el que los eunucos son más longevos y los sementales tienen una corta existencia. 
El tercer tipo: es cuando se encuentran el elefante y la oveja, y el carnero y la elefanta. En ambos, aumenta el daño, no es coincidente y ninguno le da placer al otro. Lo mejor es que sea rápida la separación y la distancia entre ellos (al-Shayzarī, 2020: 81).

\section{CONCLUSIONES}

Sobre los textos fisiognómicos en general se ha llegado a afirmar que su utilidad principal en la sociedad es la de servir a compradores y vendedores a la hora de adquirir en el zoco una o varias esclavas. De hecho, a lo largo de nuestro estudio hacemos mención de esta percepción. Sin embargo y aunque en ciertos tratados de hisba o jurídicos, por ejemplo, se explicite el acto lícito de compra-venta de esclavas, sus precios y condiciones, no hallamos afirmaciones evidentes en las fuentes que clarifiquen esta conjunción en la sociedad árabe e islámica. Así pues, sería más plausible señalar que la fisiognomía, como literatura, conforma otro modo narrativo más para relegar a las mujeres a un segundo plano, solo que, en este caso, los tratadistas emplean esta erudición como si de un texto empírico se tratase, cosa que no ocurre en otro tipo de narraciones, como bien podría ser la de tipo humorístico.

Con todo esto, la narrativa erótico-fisiognómica no tiene por objetivo perfeccionar las relaciones sexuales o la vida conyugal, como bien pudiera entenderse en un tratado de afrodisíacos o de posturas coitales. El motivo no es otro que cosificar el cuerpo de las mujeres y secundarlas como objeto pasivo en función del deseo masculino. Por esta razón, es posible que los textos fisiognómicos sirvieran como texto de asueto en el ámbito áulico para entretener a reyes y visires en sus veladas. De todo esto se deduce que el estudio de la fisiognomía femenina, al contrario de la masculina, se determina por su debilidad física y mental. Las mujeres son sometidas a la procreación y no se les concede espacio para la reflexión.

RECIBIDO: octubre 2020; ACEPTADO: febrero 2021.

\section{REFERENCIAS BIBLIOGRÁFICAS}

Álvarez de Morales, Camilo (1998): «El cuerpo humano en la medicina árabe medieval. Consideraciones generales sobre la anatomía», en E. GARCía SÁNCHEZ (ed.), Ciencias de la naturaleza en Al-Andalus: textos y estudios, 5, Consejo Superior de Investigaciones Científicas, Granada, pp. 121-135.

ANAWATI, George (1976): «Classification des sciences et structure des summae chez les auteurs musulmans", Revue des Études Islamiques 44: 64-65.

Armstrong, A. MacC. (1958): «The Methods of Greek Physiognomists», Greece and Rome 5 (1): 52-56.

BouHdibA, Abdelwahab (1980): La sexualidad en el Islam, Monte Ávila, Caracas.

Cadden, Joan (1993): Meanings of Sex Difference in the Middle Ages. Medicine, Science and Culture, Cambridge University Press, Cambridge.

Corán (1973): Edición de Juan Vernet, Editorial Planeta, Barcelona. 
GHERSETTI, Antonella (1994): «Una tabella di fisiognómica nel qabs al-anwār wa bahgat al-asrār attribuito a Ibn Arabi», Quaderni di Studi Arabi 12: 15-47.

GHERSETTI, Antonella (1999): Kitäb Aristạtạalis al-faylasüffí l-firäsa nella traduzione di Hunayn B. Is hạqu, Quaderni di Studi Arabi, Studi e Testi, 4, Venezia.

GHersetTI, Antonella (2007): «The Semiotic Paradigm: Physiognomy and Medicine in Arabic Culture», en S. SWAIN (ed.), Seeing the Face, Seeing the Soul: Polemon's Physiognomy from Classical Antiquity to Medieval Islam, 6, Oxford University Press, Oxford, pp. 281-308.

GHERSETTI, Antonella (2018): «The representation of Slave Girls in a Physiognomic Text of the Fourteenth Century», Mamluk Studies Review 21: 21-45.

JALĪFA, Ḥāŷŷȳ (1964): Kašf al-z̧unūn 'an asāmì l-kutub wa-l-funūn, 4, ed. G. Flügel, New York.

LUCENA, Miguel Ángel (2019): La sexualidad árabe e islámica medieval a través de la traducción del Kitāb al-ị̂āh fì asrär al-nikāh de al-Šayzarī, Universidad de Granada [Tesis doctoral].

Mourad, Youssef (1939): La physionomie arabe et le Kitäb al-firāsa de Fakhr al-Din al-Räzì, Paris.

AL-Neimi, Salwa (2012): El sabor de la miel, trad. Myriam Fraile, Emecé, Barcelona.

AL-ŠAYZARİ (2020): El Manifiesto de los secretos del coito: un manual árabe de afrodisíacos, trad. Miguel Ángel LuCena Romero, UCOpress y Editorial UCA, Córdoba y Cádiz.

Tena, Pedro (2008): «Mujer y cuerpo en al-Andalus», Studia Historica, Historia Medieval 26: 45-61.

AL-ŶĀHIIZ (1980): Risāla al-qiyān, ed. y trad. A. BEESTON, The Spistle of Singing-Girls by Ŷăhiz, Aris and Philipps, Warminster.

AL-ŶĀHIZZ (1998): El libro de la cuadratura del círculo, trad. Pedro Buendía PérEZ, Gredos, Madrid.

AL-YÁHIZ (2018): Elogio y diatriba de cortesanas y efebos, trads. Pedro BuENDía e Ignacio GuTIÉRREZ DE TERÁn, Ediciones del Oriente y del Mediterráneo, Madrid.

YAQŪT, al-Rūmī (2007): Mưŷ̀m al-buldān, 5 vols, Dār Sāḍir, Beirut. 